\title{
A Therapeutic Oral Vaccine Candidate against Propionibacterium acnes: Immunological Evaluation Preparation and Encapsulated Sialidase-CAMP Fusion Protein
}

\section{Zargham Parvin ${ }^{1}$}

https://orcid.org/ 0000-0001-7231-6870

Mansouri Kowsar ${ }^{2}$

https://orcid.org/0000-0002-7262-8572

\author{
Salimian Jafar ${ }^{4}$ \\ https://orcid.org/0000-0002-7015-2638
}

Ahmadi $\mathrm{Ali}^{3 *}$

http://orcid.org/0000-0001-7818-4063

Amani Jafar
http://orcid.org/0000-0002-5155-4738

${ }^{1}$ Islamic Azad University, Pharmaceutical Sciences Branch, Faculty of Advanced Sciences and Technology, Department

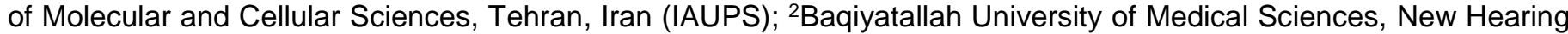
Technologies Research Center, Tehran, Iran; ${ }^{3}$ Baqiyatallah University of Medical Sciences, Systems biology and poisonings institute, Molecular Biology Research Center, Tehran, Iran; ${ }^{4}$ Baqiyatallah University of Medical Sciences, Systems biology and poisonings institute, Chemical Injuries Research Center, Tehran, Iran; ${ }^{3}$ Baqiyatallah University of Medical Sciences, Systems biology and poisonings institute, Molecular Biology Research Center, Tehran, Iran.

Received: 2019.07.18; Accepted: 2020.05.06.

*Correspondence: aliahmadi@bmsu.ac.ir; Tel.: +98-21-66056135

\section{HIGHLIGHTS}

- This study evaluated chitosan- nanoparticlated fusion protein as an acne oral vaccine.

- The Sia-CAMP protein was encapsulated in chitosan nanoparticles and induced immune response in mice.

- Although subcutaneous immunization has induced better systemic immunity, oral administration could induce both systemic and mucosal immune responses.

\begin{abstract}
Acne Vulgaris is a common skin disease caused by Propionibacterium acnes, an anaerobic microbiota of human skin that plays a vital role in the pathology of acne. The aim of this study was to prepare nanoparticles containing an acne recombinant protein and determine its ability as an oral acne vaccine in mice. The recombinant Sialidase-CAMP gene was expressed and purified in a prokaryotic host. The chitosan nanoparticles containing the recombinant protein were prepared, encapsulated, and administered by both oral and subcutaneous routes to Balb/c mice. Sera $\lg A$ and $\lg G$ and stool $\lg A$ titers were measured by ELISA, and the immunized mice were challenged against $P$. acnes. A $65 \mathrm{kDa}$ recombinant protein was confirmed by SDS-PAGE and western blot. The size and zeta potential of nanoparticles were $80 \mathrm{~nm}$ and $+18 \mathrm{mV}$, respectively. After oral immunization, the serum $\lg G$ and $\lg A$ titers were 1:3200 and 1:16, respectively, and the stool IgA titer was 1:8. In the subcutaneous route, the serum IgG titer was 1:51200. Immunized mice showed no inflammation in the ear of challenged mice. It is the first study that examines a chitosannanoparticulated acne fusion protein as an applicable acne vaccine candidate with appropriate
\end{abstract}


immunogenicity potential. Further studies are required to validate the clinical usefulness of this vaccine candidate.

Keywords: Propionibacterium acnes; Sialidase-CAMP fusion protein; chitosan nanoparticle; Acne vaccine

\section{INTRODUCTION}

Acne Vulgaris is a multifactorial disease mostly contributed by the proliferation of Gram-positive anaerobic Propionibacterium acnes. It is a common skin disease experienced by approximately $85 \%$ of individuals in 18-25 years of their life, causing both physical and psychological complications, including scar tissue, depression, anxiety, and low self-confidence (1). The main treatment for acne is retinoic acids and antibiotic treatments. Due to long-term treatment periods, antibiotic administration can disrupt the integrity of skin and intestinal resident normal microbiota as well as dominating antibiotic-resistant bacteria in patients. Also, due to various side effects such as dry skin, epistaxis, photosensitivity, increased serum lipids, and myalgia, no adequate treatment is yet available for acne $(2,3)$. In this regard, vaccine development may be considered at least as a supplementary treatment for acne disease. But, despite the importance of vaccine therapy in the clinical treatment of many diseases, limited studies have been done on acne vaccine therapy. For years, the heat-killed Propionibacterium acnes, isolated from the same acne patient's skin lesions, has been used as a traditional supplemental treatment (4). Currently, AcneVac is a commercially available vaccine in Europe as a capsule containing three standard strains of killed $P$. acnes (5). Since recombinant bacterial vaccines are a suitable alternative to traditional vaccines, some new vaccine approaches containing the bacterial surface antigens were used as an acne vaccine candidate in recent years. In some studies, Nakatsuji and coauthors proposed and evaluated the immunogenicity properties of two $P$. acnes surface proteins as the potential acne vaccine candidates $(6,7)$. They separately examined the immunogenicity of sialidase enzyme (a $502 \mathrm{kDa}$ cell wall attached protein of $P$. acnes) and CAMP factor (a $267 \mathrm{kDa}$ hemolytic protein of $P$. acnes) as subcutaneous vaccine candidates, demonstrating their capacity to reduce bacterialinduced inflammation in vivo that can neutralize $P$. acnes in vitro $(6,7)$. In a previous study, we in silico designed a chimeric protein vaccine candidate that contained immunogenic parts of the sialidase and CAMP factor linked by four repeated EAAAK linkers (the Sia-CAMP fusion protein) (8). We showed that this fusion protein could make a strong immune response by immunoinformatic evaluation. In this study, we tried to investigate the immunogenicity potential of this fusion protein in a mice model by oral immunization. For years, oral immunization with vaccines has been examined against bacterial infections. Although being potentially immunologic, cost-benefit, applicable, and painless, only a few mucosal vaccines are available. Nanoparticulation with chitosan and other polysaccharides is the main strategy used for oral vaccine development. Chitosan, a natural linear polysaccharide made of D-glucosamine and N-acetyl-D-glucosamine units, is a suitable biopolymer for various biomedical applications including nonvaccine development (9). Regarding the advantages of oral vaccine, we aimed to express, purify, and immunologically evaluate the chitosan nanoparticlated Sia-CAMP fusion protein in mice model through the oral and injection routes as a therapeutic acne vaccine candidate.

\section{MATERIAL AND METHODS}

\section{Mice and ethics statement}

The 6-8-week-old female BALB/c mice were purchased from Razi Institute (Karaj, Iran) and maintained in polypropylene cages at $20-22^{\circ} \mathrm{C}, 12-\mathrm{h}$ light/dark cycles. All experimental procedures on animals were approved by the local ethical committee of Baqiyatallah University of Medical Sciences.

\section{Expression, purification, and western blotting}

In the previous study, a synthetic construct containing the C-terminal amino acids of the sialidase and $\mathrm{N}$-terminal amino acids of the Camp factor was designed, and linked by a rigid EAAAK linker. Then, the recombinant Sia-CAMP gene sequence along with the EcoR1 and Hind III restriction sites sequences at 3' and $5^{\prime}$ ends of the construct was synthesized with codon optimization for a prokaryotic host (8). After cloning in pET28a (+), the "pET28a /Sia-CAMP" construct was transformed and expressed in the E. coli BL21 DE3 host. For gene expression induction, IPTG $(1 \mathrm{mM})$ was added to the nightly cultured bacteria containing kanamycin $(20 \mu \mathrm{g} / \mathrm{mL})$ (10). After centrifugation (6000 RPM, 25min), the bacteria were sonicated, centrifuged, and the pellet was dissolved in lysis buffer ( $8 \mathrm{M}$ urea, $0.1 \mathrm{NaH} 2 \mathrm{PO} 4$, and $0.01 \mathrm{M}$ Tris). After centrifugation, the supernatant was run on $12 \%$ SDS-PAGE (11). For protein purification, the cell lysate supernatant was 
loaded on the Nickel-nitrilotriacetic acid (Ni-NTA) affinity column using a buffer containing imidazole $500 \mathrm{mM}$ as the eluting buffer. Finally, the protein concentration was determined by Bradford assay, and western blotting was done by standard protocols $(10,11)$.

\section{Preparation of chitosan nanoparticles}

Chitosan nanoparticles were prepared by ionic gelation. A solution of $2 \mathrm{mg} / \mathrm{mL}$ chitosan in $2 \%$ acetic acid and $1 \mathrm{mg} / \mathrm{mL}$ sodium polyphosphate solution were prepared. The sodium tripolyphosphate solution $(5 \mathrm{~mL})$ was added dropwise to $5.7 \mathrm{~mL}$ of chitosan solution containing the antigen, centrifuged for 40 minutes at $13,000 \mathrm{rpm}$, and the supernatant was separated $(12,13)$. The morphological properties of Sia-CAMP recombinant protein-loaded chitosan nanoparticles were examined using a scanning electron microscope (SEM). Also, the loading efficiency was calculated based on the difference in the amount of the initial protein added to the chitosan solution and the final amount of the residue in the supernatant through the following formula:

$$
\text { LE (Loading efficiency) }=\frac{\text { Total amount of protein-Free protein }}{\text { Total amount of protein }} \times 100
$$

Also, the size of nanoparticles was determined using the DLS device model Malvern.

\section{Mice Immunization with the antigen-loaded chitosan nanoparticles}

Three mice groups (5mice/group) were used to compare the systemic and mucosal immune responses against the Sia-CAMP fusion protein. For the systemic immune response evaluation, $20 \mu \mathrm{g}$ of Sia-CAMP loaded nanoparticles were injected subcutaneously into the first mice group, followed by three booster doses within two weeks intervals. For the assessment of mucosal immune responses, $100 \mu \mathrm{g}$ of Sia-CAMP loaded nanoparticles were gavaged to the second group with an additional three booster doses within two weeks intervals. As a control group, the third group was orally received nanoparticle loaded Bovine Serum Albumin in the same manner. The groups of immunized and control mice are shown in Table 1.

\section{Mice Immunization with heat-inactivated P. acnes}

To compare the immunization of nanoparticles loaded with the fusion protein as well as the heatinactivated $P$. acnes, another group of five mice were immunized with heat-inactivated $P$. acnes. Freezedried $P$. acnes (PTCC 6919) was prepared from the Pasteur Institute of Iran (Tehran, Iran) and re-confirmed with standard microbiological methods. To prepare the inactivated $P$. acnes, a PBS dissolved colony suspension $\left(10^{7} \mathrm{CFU} / \mathrm{mL}\right)$ was centrifuged, washed three times, and heated at $65^{\circ} \mathrm{C}$ for $45 \mathrm{~min}$. After treatment, no bacterial growth was confirmed by culture in specific culture media. Then, the suspension was subcutaneously injected with the complete Freund's adjuvant (for the first injection) and incomplete Freund's adjuvant (three boosters; 2 weeks interval) into mice. The groups of immunized and control mice are shown in Table 1.

\section{Determination of the antibody titer by indirect ELISA}

Two weeks after each booster, peripheral blood was obtained from the retro-orbital plexus of all immunized and control mice groups. The serum titers of $\lg G$ and $\lg A$ against the Sia-CAMP fusion protein were measured by indirect ELISA using the standard protocol (14). Briefly, 96-well ELISA plates (NUNC, Denmark) were coated with the Sia-Camp fusion protein $(1 \mu \mathrm{g} / 100 \mu \mathrm{l} /$ well $)$ in the carbonate-bicarbonate buffer $\left(37^{\circ} \mathrm{C}, 2 \mathrm{~h}\right)$. The plates were washed with PBST $(4 \mathrm{X})$ and blocked with skim milk solution $2 \%(\mathrm{w} / \mathrm{v})$ in PBST. Then, the plates were incubated with a serial dilution of 1:100 to 1:100000 for $\lg G$ and 1:2 to 1:32 for $\lg A$ $\left(37^{\circ} \mathrm{C}, 1 \mathrm{~h}\right.$ ). Finally, anti-mouse IgG and IgA- HRP conjugated antibodies (Sigma, USA) were subjected to the plates. The sera collected from mice before immunization was used as negative control. For secretary IgA determination, fecal samples were collected two weeks after each booster from orally immunized mice. The PBS buffer containing cocktail protease inhibitor (Sigma, USA) was added to mice feces, roughly vortexed, and centrifuged $14000 \mathrm{rpm}$ for $10 \mathrm{~min}$. Finally, the supernatant was subjected to the Sia-CAMP protein-coated plate (15).

\section{Challenge}

For checking the immunization, $10^{7} \mathrm{CFU}$ of $P$. acnes was diluted in PBS buffer and subcutaneously injected into one of the ears of all immunized and control mice groups. Besides, PBS was injected into the 
other ear, as the injection control. Then, during 40 days, the thickness and inflammation of the ear were recorded by micro-caliper until the swelling of the ear almost dropped.

\section{Statistical analysis}

The statistical analyses were carried out with SPSS statistical software (version 20). Data obtained from the antibody detection and also protection was analyzed using the two-way analysis of variance (ANOVA). $P$ values $<0.05$ were considered statistically significant.

\section{RESULTS}

\section{Expression and purification of the recombinant protein}

After induction of the chimeric gene in optimized condition ( $1 \mathrm{mM}$ IPTG after $3 \mathrm{~h}$ at $37^{\circ} \mathrm{C}$ ), the protein content was run on $12 \%$ SDS-PAGE showing a complete expression of Sia- CAMP fusion (Figure 1). The majority of the fusion protein was expressed as a soluble protein, and following Ni-NTA affinity chromatography, the Sia-CAMP fusion was purified with a weight of $65 \mathrm{KD}$ with high purity at $500 \mathrm{mM}$ imidazole elution. Then, the protein expression was confirmed by western blotting due to having a His-tag sequence, with the desired band sited in the correct weight position with no other bands in the column control (Figure 2).

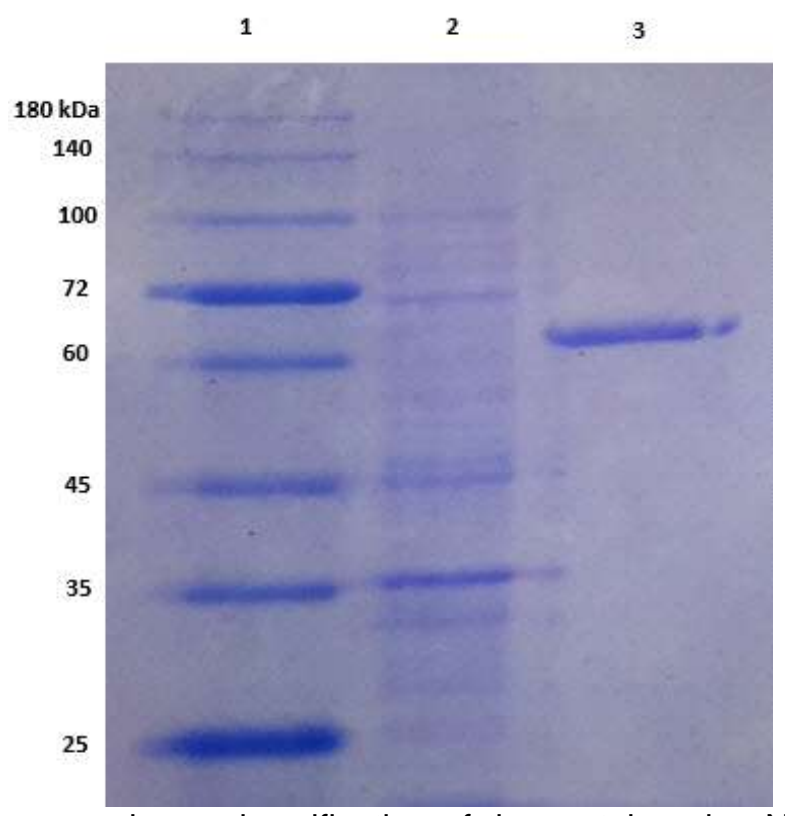

Figure 1. Gel electrophoresis after expression and purification of the protein using NI-NTA. Lane 1: protein weight marker; lane 2: Control or non-induced sample; lane 3: wash column with 500mM imidazole.

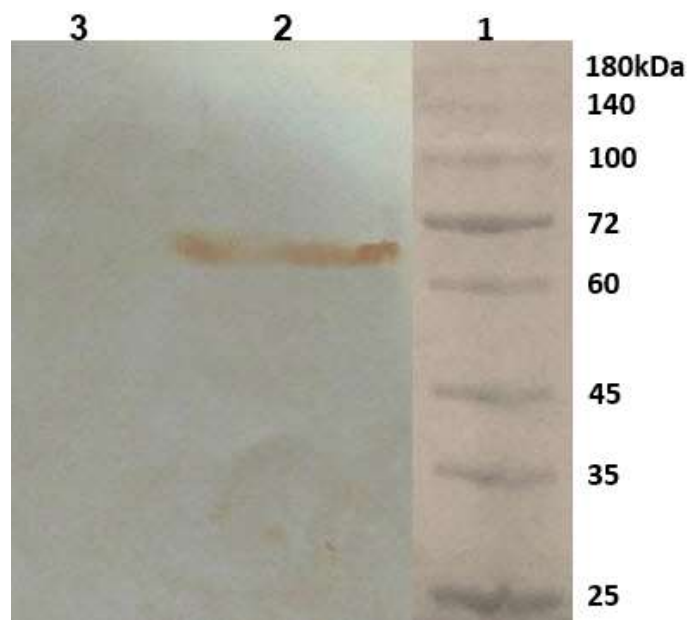

Figure 2. Confirmation of the recombinant protein by Western blotting. Column 1: Protein Molecular Marker; Column 2: The desired protein; Column 3: Control Sample (No IPTG). 


\section{Preparation of chitosan nanoparticles and protein loading rate}

After the chitosan nanoparticle preparation by the ion gelation method and size measurement, the result of DLS indicated the production of chitosan nanoparticles with an average size of $85 \mathrm{~nm}$. Zeta potential of the nanoparticles containing the recombinant protein was $+18 \mathrm{mV}$. Also, after optimizing nanoparticles preparation, the loading rate was estimated at $88 \%$. Besides, as shown in Figure 3, analyzing SEM images showed that the average diameter of the produced monodispersed spherical nanoparticles containing SiaCAMP protein was found to be about $90 \mathrm{~nm}$ (Figure 3).

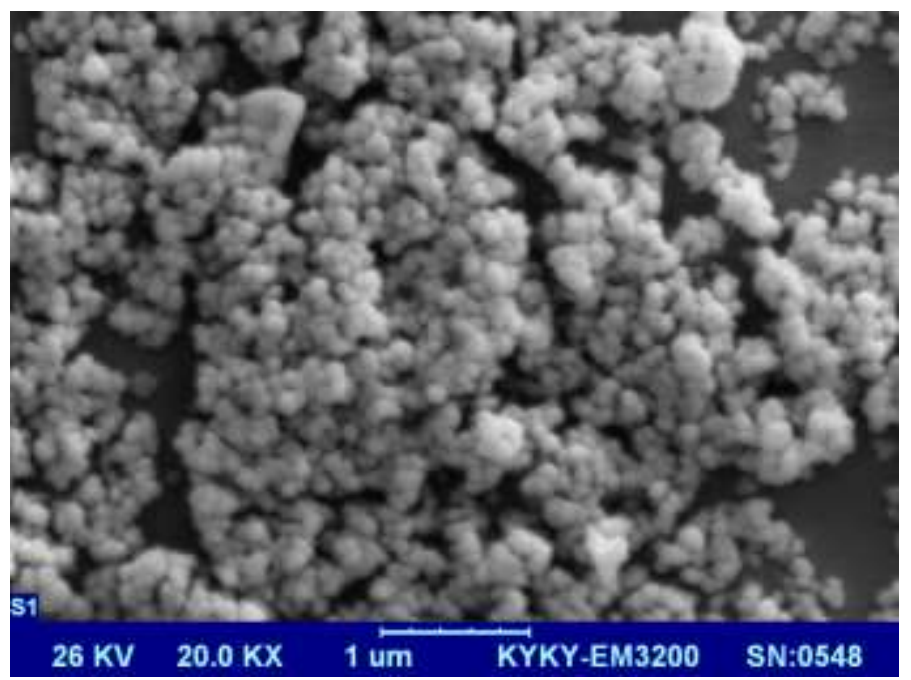

Figure 3. SEM images of Sia-CAMP fusion protein loaded chitosan nanoparticles.

\section{Measurement of antibody titer}

The IgG antibody titers were measured in serum samples for all groups. Also, the $\lg A$ antibody was tittered in both serum and stool samples of immunized and control mice groups. When the protein was injected with chitosan nanoparticles, after each booster, the $\operatorname{lgG}$ antibody titer increased significantly, from 1:6400 to 1: 12800 in the first booster, and finally to 1:51200 in the second booster. Chitosan nanoparticles have also been shown to be an appropriate adjuvant and could induce high titers of systemic lgG in mice (Figure 4, A). In comparison, the mucosal administration of chitosan nanoparticulated protein could pass the protein through the intestinal barrier and induced a strong systemic immune response of $\operatorname{lgG}$ with a titer of 1: 32000 (Figure 4, B). Also, it stimulated the mucosal immune system and increased serum IgA titers to 1:32 (Figure 4, D). Measurements of secretory $\lg A$ in feces also showed the induction of mucosal responses in mice up to the 1:8 titer (Figure 4, E). In contrast, the heat-inactivated $P$. acnes, which was injected with incomplete and complete Freund's adjuvant, induced only a systemic immune response, and IgG titers reached 1:102400 in the third booster (Figure 4,C). Furthermore, the mean IgG titer was compared between injection and oral administration routes of the nanoparticulated fusion protein with that of heat-inactivated bacteria (Figure 5). The mice immunized with heat-inactivated $P$. acnes showed higher IgG titers rather than the control group $(p<0.003)$. Also, in the mice group immunized with antigen-loaded nanoparticles, although the $\lg G$ titers were lower than mice immunized with heat-inactivated $P$. acnes, they had a significantly higher lgG titer against the control group $(p<0.003)$. The nanochitosan could show an adjuvanticity property during the injection and oral administration routes of the fusion protein and induced systemic immune responses in mice. 
(C)
(A)
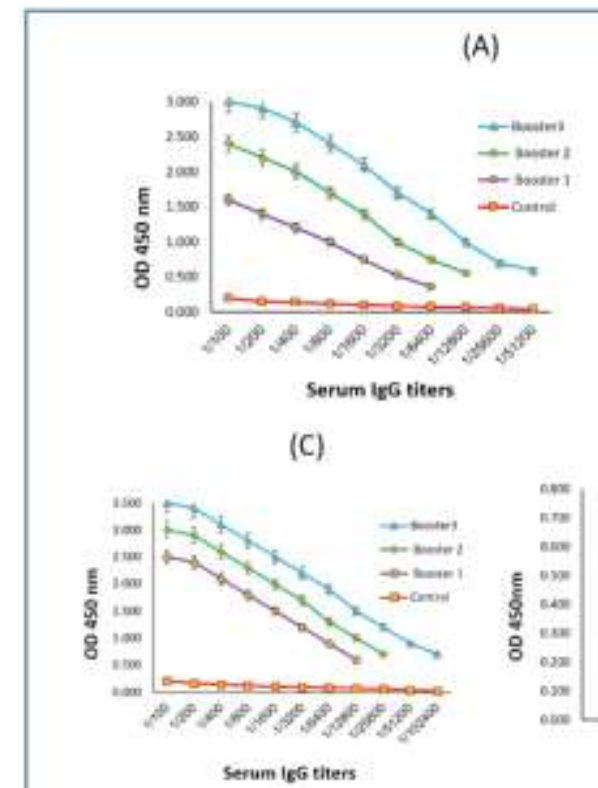

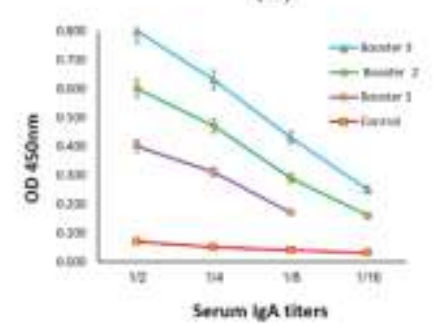

(B)

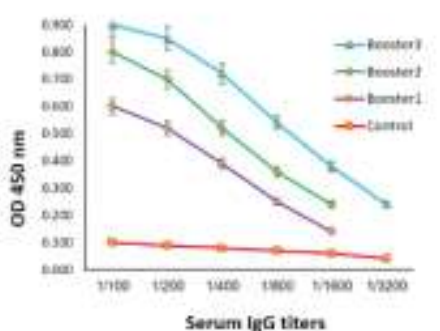

(D)

(E)

Figure 4. $\lg G$ and $\lg A$ antibody titers against Sia-CAMP fusion protein, and heat-inactivated $P$. acnes that were measured in Balb/c mice. (A) Serum IgG antibody titer after injection of antigen-loaded nanoparticles. (Test samples include antigen-loaded nanoparticles and control samples include antigen-free nanoparticles). (B) Serum IgG antibody titer after oral administration of the antigen-loaded nanoparticles. (The test samples included antigen-loaded nanoparticles and control samples include antigen-free nanoparticles). (C) Serum IgG antibody titer after the standard vaccination with the heat-inactivated bacteria. (D) Serum IgA antibody titer after oral administration of antigen-loaded nanoparticles. (E) Secretory IgA antibody titer after oral administration of antigen-loaded nanoparticles. Data expressed as mean analyzed sera/mice group. Values are significantly different from the control at $p<0.001$.

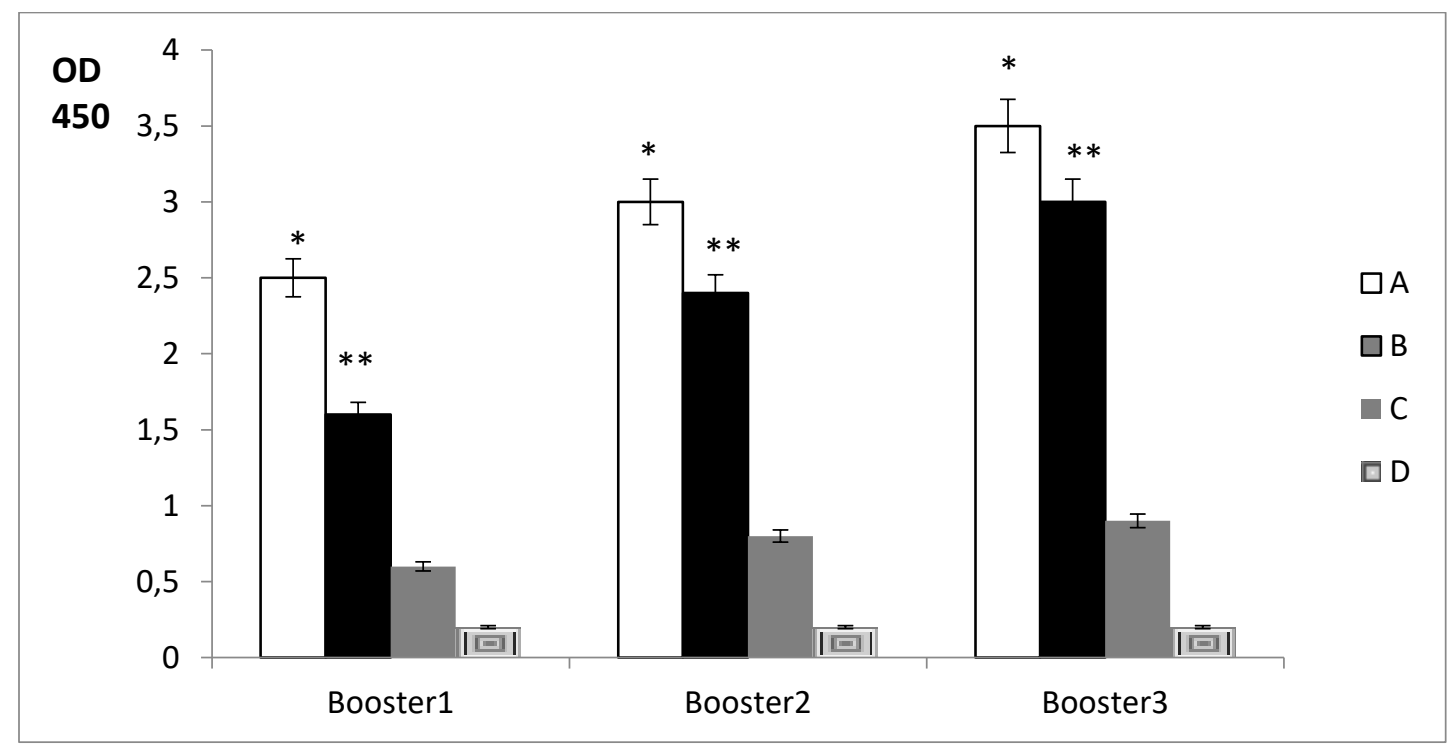

Figure 5. Comparison of serum IgG titers (dilution 1:100). The column includes heat-inactivated $P$. acnes subcutaneous injection (A), antigen-loaded nanoparticles subcutaneous injection (B), antigen-loaded nanoparticles oral administration (C), antigen-free nanoparticles oral administration (control) (D). Data expressed as mean analyzed sera/mice group. * and $^{* *}$ : Values significantly different from the control at $p<0.003$.

\section{The challenge result}

After two months of the last immunization, the protection level of the fusion protein was determined by challenging immunized mice with live $P$. acnes. The results showed that there is no inflammation, induration, and redness in the ear of both immunized mice groups, but the inflammation was induced in $50 \%$ of the control mice group. The results of the challenge with $10^{7} \mathrm{CFU}$ of $P$. acnes are shown in Table 2. 
Table 2. The mice group challenged with 107CFU of $P$. acnes injected into mice ear.
Mice group
Ear thickness and Inflammation

$\begin{array}{lc}\text { Nanoparticulated fusion protein (injection) } & \text { No } \\ \text { Nanoparticulated fusion protein (oral) } & \text { No } \\ \text { Killed P. acnes (injection) } & \text { No } \\ \text { Control } & \text { Yes (50\%) }\end{array}$

\section{DISCUSSION}

Deficiencies and problems due to current therapeutic managements for acne disease, especially in severe acne vulgaris cases warrant developing new therapeutic strategies, including vaccine therapy. Although some efforts to achieve a therapeutic $P$. acnes vaccine, not such a satisfactory vaccine has been introduced to date, maybe due to the multifactorial nature of acne disease as well as various heterogeneous bacterial virulence factors $(16,17)$. Accordingly, the present study sought to introduce a chimeric protein antigen encapsulated in chitosan nanoparticle being able to induce local as well as systemic immune responses against $P$. acnes. Chimeric vaccines, with utmost attention in infectious disease prevention, have some benefits such as increased safety, cost-benefit, and less antigenic competition, making them appropriate vaccine candidates in may studies $(18,19)$. Therefore, in our recent bio-computational study, we introduced a Sialidase-CAMP chimeric vaccine candidate, based on two separated Nakatsuji studies $(6,7)$, and postulated that it could be able to induce immune responses (8). Now, in the present study, this chimeric construct was cloned, expressed, and used in a nanoparticle formulation. We aimed to evaluate the immunogenicity of this chimeric construct through oral administration. Oral vaccines, as a non-invasive route, remove disadvantages associated with the injection route, including pain, discomfort, and safety biohazard. Chitosan is a highly biocompatible, bioavailable, biodegradable, non-toxic material. It has antimicrobial properties as well as good adhesion strength, making it be approved as a Generally Recognized as Safe (GRAS) material, according to United States Food and Drug Administration (USFDA) (20). Accordingly, we used chitosan nanoparticles for oral administration to protect Sia-CAMP protein from enzymatic degradation of the gastrointestinal tract (21-23). Also, the ion gelation method was used to prepare chitosan nanospheres. The advantages of this method include the absence of harmful organic solvent, high encapsulation, and also controlled releasing (24). In addition, the chitosan solution with pH 5.5 was used for making chitosan nanospheres, because the chitosan molecule is more open at this $\mathrm{pH}$ and have more active sites for the formation of hydrogen bonds with protein molecules (25). Using $1 \mathrm{mg} / \mathrm{mL}$ chitosan for nanoparticulation, the size of the nanoparticle was about $80 \mathrm{~nm}$, with a zeta potential of $+18 \mathrm{mV}$, and a loading rate of $88 \%$. Other studies have indicated a maximum of $100 \mathrm{~nm}$ as the optimum size of nanoparticles, making the antigens easily transfer from the injection site to the lymph nodes. In the innate immune system, antigen-presenting cells (APCs) such as dendritic cells and macrophages as the first line of immune defense cells could more efficiently uptake the nanoparticle below the $100 \mathrm{~nm}$ particle size. The size, shape, and elasticity of particle affect the immunogenicity properties and potential. Particles smaller than $40 \mathrm{~nm}$ induce cell-mediated immunity (TH1 mediated responses), while particles equal or larger than 100-nanometer could stimulate humoral immunity and antibody production (26). In comparison, Bagheripour and coauthors used chitosan nanoparticles as a carrier for the recombinant BoNT/E binding protein by the oral route. In this study, the size of the nanoparticle and the loading efficiency rate was $285 \mathrm{~nm}$ and $91 \%$, respectively (23). Jesus and coauthors used Poly- $\varepsilon$-caprolactone/chitosan nanoparticles as carriers of the hepatitis $B$ antigen recombinant protein. The size of the nanoparticle and the zeta potential was $201 \mathrm{~nm}$ and $+18.6 \mathrm{mV}$, respectively, with a loading rate of 96 percent (27). Zolfagharnia and coauthors used nanoparticles composed of thiolated methylated pyridinyl chitosan as a new strategy for buccal drug delivery of insulin. The size of the nanoparticles and the zeta potential were $268 \mathrm{~nm}$ and $+28.3 \mathrm{mV}$, respectively, and a loading rate of $91+2.6$ percent (28). Nesalin and coauthors used chitosan nanoparticles with various concentrations as a carrier for the zidovudine. The size of the nanoparticles and the zeta potential were approximately $342-468 \mathrm{~nm}$ and 20.4 to $37.08 \mathrm{mV}$, respectively (13). In this study, two routes (oral and subcutaneous routes) were used for 
administration. In both groups, immunized Balb/c mice showed high titers of serum IgG. For injected mice, the raised antibody titer suggested that nanoparticles can act as an adjuvant to induce immune responses. In orally immunized mice, nanoparticulation could protect against gastrointestinal degradation. The lgG titers were 1: 51200 and 1:3200 in subcutaneous and oral administration, respectively. Also, the vaccine mice group immunized with inactivated bacteria gave an IgG titer of 1:102400. In 2008, Nakatsuji and coauthors intranasally immunized mice with heat-killed $P$. acnes and found that the most antibodies are induced against two major antigens of $P$. acnes, including sialidase and CAMP factor (7). Later in another study, they showed that the mice had low protection against $P$. acnes when immunized with inactivated whole bacteria. They reported that sialidase was immunogenic in mice vaccinated with a recombinant sialidase (7). In the Nakatsuji study in 2011, the recombinant CAMP factor was administrated by both intranasal and subcutaneous routes. Twenty-one days after immunization, the lgG titer was higher than 100,000 in the sera of immunized mice. According to two Nakatsuji studies, we aim to test whether the recombinant Sia-CAMP encapsulated fusion protein could elicit the mucosal immunity against $P$. acnes. In the present study, the lgG titer of the recombinant Sia-CAMP injected subcutaneously was approximately equal to the anti-CAMP IgG titer in the Nakatsuji study. Of note, they used the CAMP factor in conjunction with Freund's adjuvant (6), and in the present study, the chitosan nanoparticle played the role of the adjuvant. However, in the oral administration of the Sia-CAMP protein, the $\operatorname{lgG}$ antibody titer was lower. Also, in orally immunized mice, $\lg A$ titers in serum and fecal samples were 1:16 and 1:8, respectively, suggesting inducing the mucosal responses. The stool $\lg$ A titer was lower than the serum, which may be due to proteolytic degradation of antibodies before adding anti-protease (29). Unfortunately, no other study is available for the comparison of our data. In this study, to determine the immunity induced by recombinant Sia-CAMP protein, the ears of the vaccinated mice were subcutaneously challenged with live $P$. acnes. By examining the mice visually, ear inflammation was seen in $50 \%$ of the control group. The live bacteria injected into the mice group, immunized with the recombinant protein, did not affect the mice ears, and inflammation was not observed. Therefore, it seems that this recombinant protein is a suitable vaccine candidate, though it needs more clinical and immunological evaluations. In 2008, Nakatsuji and coauthors challenged the ear of the vaccinated mice with heat-killed $P$. acnes intradermally with living $P$. acnes. They showed that the bacterial progression might be suppressed by using inactivated $P$. acnes-based vaccines. This vaccination could also facilitate the recovery of $P$. acnesinduced inflammation (7). In another study in 2008, Nakatsuji and coauthors elicited a detectable antibody by sialidase-immunized mice. These antibodies could neutralize the $P$. acnes cytotoxicity in vitro and induce the production of $P$. acnes interleukin-8 (IL-8) in human sebocytes. Also, this treatment blocked the increase in ear thickness and also prevented the release of pro-inflammatory macrophage inflammatory protein (MIP2) cytokine. A study by Wang and coauthors showed that mutations in the CAMP factor could significantly decrease the bacterial colonization and subsequent inflammation in mice. In addition, vaccination with CAMP factor could induce the production of MIP-2 (macrophage inflammatory protein-2) in mice. Using ex vivo acne model, they concluded that $P$. acnes CAMP factor is an essential source of inflammation in acne vulgaris and suggested that CAMP factor may be considered as a candidate target in acne vaccination (30). In another study by Agak, they showed that the functions of T helper 17 (Th17) cells are different between healthy skins versus acne associated $P$. acnes strains. They observed that acne-associated $P$. acnes strains induce higher IL-17 levels than healthy skin strains due to inducing different phenotype and function of Th17 cells. They finally suggested that acne disease-causing strains may generate Th17 cells that may contribute more efficiently to acne pathogenesis (31). Interestingly, recent work revealed that there are different phylogenetic of $P$. acnes strains, and these phylotypes can induce different immunological responses. In this regard, Yang and coauthors reported that acne-associated $P$. acnes phylotypes induced 2-3 folds higher levels of IFN- $\gamma$ and IL-17 in peripheral blood mononuclear cells compared with healthy phylotypes. Meanwhile, healthy skin $P$. acnes strains are associated with induced 2-4 folds higher levels of IL-10 (32). This studies more emphases on immunological complexity of acne vulgaris disease.

\section{CONCLUSION}

It is the first study to design and evaluate chitosan- nanoparticlated fusion protein as an acne oral vaccine. Our results indicate that the Sia-CAMP fusion protein was expressed correctly and was encapsulated successfully in the chitosan nanoparticles, and has been able to induce a systemic and topical immune response in mice model. Although subcutaneous immunization has induced better systemic immunity, oral administration can induce as the therapeutic vaccine both systemic and mucosal immune responses. It seems that this approach can be a supplemental therapeutic method-along with other medications- for the management of severe acne diseases. 
Funding: This research received no external funding.

Acknowledgments: The authors thank dear colleagues from the research laboratory at Baqhiyatallah University of Medical Sciences who have contributed to this research.

Conflicts of Interest: The authors have no conflict of interest.

\section{REFERENCES}

1. Seth V, Mishra A. Acne vulgaris management: what's new and what's still true. International Journal of Advances in Medicine. 2015;2(1):1.

2. Simonart T. Newer approaches to the treatment of acne vulgaris. American journal of clinical dermatology. 2012;13(6):357-64.

3. Lukaviciute L, Navickas P, Navickas A, Grigaitiene J, Ganceviciene R, Zouboulis C. Quality of life, anxiety prevalence, depression symptomatology and suicidal ideation among acne patients in Lithuania. Journal of the European Academy of Dermatology and Venereology. 2017;31(11):1900-6.

4. Yen T, Yuan C-Y, Huang C-M. Current status of acne vaccines. Expert Review of Dermatology. 2010;5(5):561-6.

5. Bioveta. Bacterial lysate from bacterial strains of most frequent causative agents of skin diseases Named ACNEVAC 2016 [Nov 22, 2017]. Available from: http://www.bioveta.eu/en/products/human-products/acnevac4.html.

6. Nakatsuji T, De-chu CT, Zhang L, Gallo RL, Huang C-M. Propionibacterium acnes CAMP factor and host acid sphingomyelinase contribute to bacterial virulence: potential targets for inflammatory acne treatment. PloS one. 2011;6(4):e14797.

7. Nakatsuji T, Liu Y-T, Huang C-P, Gallo RL, Huang C-M. Antibodies elicited by inactivated propionibacterium acnesbased vaccines exert protective immunity and attenuate the IL-8 production in human sebocytes: relevance to therapy for acne vulgaris. Journal of Investigative Dermatology. 2008;128(10):2451-7.

8. Ahmadi A, Farhadi E, Salimian J, Amani J. Designing a vaccine therapy candidate against Propionibacterium acnes: a bioinformatics approach. Molecular Genetics, Microbiology and Virology. 2016;31(3):178-86.

9. Biao L, Tan S, Wang Y, Guo X, Fu Y, Xu F, et al. Synthesis, characterization and antibacterial study on the chitosanfunctionalized Ag nanoparticles. Materials Science and Engineering: C. 2017;76:73-80.

10. Amani J, Salmanian AH, Rafati S, Mousavi SL. Immunogenic properties of chimeric protein from espA, eae and tir genes of Escherichia coli O157: H7. Vaccine. 2010;28(42):6923-9.

11. Khaledi A, Bahador A, Esmaeili D, Derakhshan M, Mahdavi M, Amani J, et al. Cloning, expression and purification of $\mathrm{HpaA}$ recombinant protein of Helicobacter pylori as a Vaccine candidate. Journal of Pure and Applied Microbiology. 2016;10(3):1937-42.

12. Vaezifar S, Razavi S, Golozar MA, Karbasi S, Morshed M, Kamali M. Effects of some parameters on particle size distribution of chitosan nanoparticles prepared by ionic gelation method. Journal of Cluster Science. 2013;24(3):891-903.

13. Nesalin JAJ, Smith AA. Preparation and evaluation of chitosan nanoparticles containing zidovudine. Asian J Pharm Sci. 2012;7(1):80-4.

14. Abkar M, Lotfi AS, Amani J, Eskandari K, Ramandi MF, Salimian J, et al. Survey of Omp19 immunogenicity against Brucella abortus and Brucella melitensis: influence of nanoparticulation versus traditional immunization. Veterinary research communications. 2015;39(4):217-28.

15. Amini Y, Tebianian M, Mosavari N, Fasihi Ramandi M, Ebrahimi SM, Najminejad H, et al. Development of an effective delivery system for intranasal immunization against Mycobacterium tuberculosis ESAT-6 antigen. Artificial cells, nanomedicine, and biotechnology. 2017;45(2):291-6.

16. O'Neill AM, Gallo RL. Host-microbiome interactions and recent progress into understanding the biology of acne vulgaris. Microbiome. 2018;6(1):177.

17. $\mathrm{Xu} \mathrm{H}, \mathrm{Li} \mathrm{H}$. Acne, the skin microbiome, and antibiotic treatment. American journal of clinical dermatology. 2019;20(3):335-44.

18. Sjatha F, Kuwahara M, Sudiro TM, Kameoka M, Konishi E. Evaluation of chimeric DNA vaccines consisting of premembrane and envelope genes of Japanese encephalitis and dengue viruses as a strategy for reducing induction of dengue virus infection-enhancing antibody response. Microbiology and immunology. 2014;58(2):12634.

19. Yang L, Cai C, Feng Q, Shi Y, Zuo Q, Yang H, et al. Protective efficacy of the chimeric Staphylococcus aureus vaccine candidate IC in sepsis and pneumonia models. Scientific reports. 2016;6(1):1-13.

20. Park JH, Saravanakumar G, Kim K, Kwon IC. Targeted delivery of low molecular drugs using chitosan and its derivatives. Advanced drug delivery reviews. 2010;62(1):28-41. 
21. Wang JJ, Zeng ZW, Xiao RZ, Xie T, Zhou GL, Zhan XR, et al. Recent advances of chitosan nanoparticles as drug carriers. Int J Nanomedicine. 2011;6(9):765-74.

22. Hajizade A, Ebrahimi F, Salmanian A-H, Arpanae A, Amani J. Nanoparticles in vaccine development. Journal of Applied Biotechnology Reports. 2015;1(4):pp. 125-34.

23. Bagheripour M, Ebrahimi F, Hajizadeh A, Nazariat S, Arefpour M. Preparation of Chitosan Based Botulinum Neurotoxin E Recombinant Nanovaccine and Evaluation of its Immunogenicity as Oral \& Intradermal Route in Mice Journal Rafsanjan University Med Sci. 2016;14(11):932-8.

24. Zaharoff DA, Rogers CJ, Hance KW, Schlom J, Greiner JW. Chitosan solution enhances both humoral and cellmediated immune responses to subcutaneous vaccination. Vaccine. 2007;25(11):2085-94.

25. Ibraheem D, Elaissari A, Fessi $H$. Administration strategies for proteins and peptides. International journal of pharmaceutics. 2014;477(1-2):578-89.

26. Benne N, van Duijn J, Kuiper J, Jiskoot W, Slütter B. Orchestrating immune responses: How size, shape and rigidity affect the immunogenicity of particulate vaccines. Journal of Controlled Release. 2016;234:124-34.

27. Jesus S, Soares E, Borchard G, Borges O. Poly- $\varepsilon$-caprolactone/chitosan nanoparticles provide strong adjuvant effect for hepatitis B antigen. Nanomedicine. 2017;12(19):2335-48.

28. Zolfagharnia B, Mortazavian E, Kaviani D, Rafiee-Tehrani M. Preparation and evaluation of nanoparticles composed of thiolated methylated pyridinyl chitosan as a new strategy for bucal drug delivery of insulin. Nanomedicine Journal. 2017;4(2):83-8.

29. Brandtzaeg P. Secretory IgA: designed for anti-microbial defense. Frontiers in immunology. 2013;4:222.

30. Wang Y, Hata TR, Tong YL, Kao M-S, Zouboulis CC, Gallo RL, et al. The anti-inflammatory activities of Propionibacterium acnes CAMP factor-targeted acne vaccines. Journal of Investigative Dermatology. 2018;138(11):2355-64.

31. Agak GW, Kao S, Ouyang K, Qin M, Moon D, Butt A, et al. Phenotype and antimicrobial activity of Th17 cells induced by Propionibacterium acnes strains associated with healthy and acne skin. Journal of Investigative Dermatology. 2018;138(2):316-24.

32. Yu Y, Champer J, Agak GW, Kao S, Modlin RL, Kim J. Different Propionibacterium acnes phylotypes induce distinct immune responses and express unique surface and secreted proteomes. Journal of Investigative Dermatology. 2016;136(11):2221-8.

(c) 2020 by the authors. Submitted for possible open access publication under the terms and conditions of the Creative Commons Attribution (CC BY NC) license (https://creativecommons.org/licenses/by-nc/4.0/). 